Novel Separation of Actinides

R. Mariella

February 28, 2011 
This document was prepared as an account of work sponsored by an agency of the United States government. Neither the United States government nor Lawrence Livermore National Security, LLC, nor any of their employees makes any warranty, expressed or implied, or assumes any legal liability or responsibility for the accuracy, completeness, or usefulness of any information, apparatus, product, or process disclosed, or represents that its use would not infringe privately owned rights. Reference herein to any specific commercial product, process, or service by trade name, trademark, manufacturer, or otherwise does not necessarily constitute or imply its endorsement, recommendation, or favoring by the United States government or Lawrence Livermore National Security, LLC. The views and opinions of authors expressed herein do not necessarily state or reflect those of the United States government or Lawrence Livermore National Security, LLC, and shall not be used for advertising or product endorsement purposes.

This work performed under the auspices of the U.S. Department of Energy by Lawrence Livermore National Laboratory under Contract DE-AC52-07NA27344. 


\title{
Novel Separation of Actinides
}

\author{
Raymond Mariella
}

10-FS-001

\begin{abstract}
The separation of actinides and other elements of interest for nuclear forensics and threat reduction is currently performed using decades-old chemistries and ion-exchange columns. We propose to determine the technical feasibility of a novel method for separating actinide ions in solution. This method is based upon isotachophoresis (ITP), which has been applied in the purification of pharmaceuticals and other biochemical applications. This technique has the potential to separate inorganic ions more effectively than existing methods, which is key to analyzing very small samples. We will perform a quantitative assessment of the effectiveness of specific isotachophoretic approaches including predicting the physical and chemical properties, such as ion mobility, of inorganic ions under specific solvent conditions using a combination of ab initio calculations and semi-empirical methods.
\end{abstract}

We expect to obtain a thorough understanding of the analytical systems parameters under which ITP is most effective for the separation of inorganic samples, including the influence of the double layer surrounding actinide ions, the Debye length for different ions and ion complexes, and Debye-Hückel limits.

\section{Mission Relevance}

Inorganic separations are key to nuclear forensics for countering terrorism and nuclear proliferation. If found to be feasible and potentially superior to currently used separation approaches, ITP could provide the conceptual basis for an improved means to separate samples of nuclear explosion debris for nuclear forensic analysis, in support of the Laboratory's missions in homeland and national security.

\section{FY10 Accomplishments and Results}

During FY10, we conducted an extensive search and study of the literature. As a result, we (1) determined that none of the publications, which date back to the conception of what is now called ITP, reported the relative or absolute mobilities of the actinide ions; (2) identified a theoretical approach that would enable us to calculate the relevant electrophoretic mobilities; (3) determined that the most important aspect of ITP is that it discards unwanted constituents, such as salts of calcium, magnesium, aluminum, sodium, potassium, and similar ions, while trapping and purifying desired ions, such as uranium dioxide $\left(\mathrm{UO}_{2}^{++}\right) ;(4)$ determined that a key aspect of ITP is that it concentrates minor or trace elements, matching the concentration of the leading electrolyte, which makes it highly appropriate for the analysis of actinides, the starting concentrations of which can span a range of several decades; (5) determined that direct interfacing of separated actinide ions to inductively coupled plasma mass spectroscopy would be possible; and (6) determined that simple models, such as Debye-Hückel, are too limited to be of predictive value for ITP in applications in which final purified concentrations exceed $0.001 \mathrm{M}$, and that ab initio and Monte Carlo models, such as those published for $\mathrm{UO}_{2}{ }^{++}$, are therefore needed. In summary, this project's findings strongly support the use of ITP as a key step in the analysis of actinides, including pre- and post-detonation nuclear forensics; that it is the only alternative to time-tested but highly labor-intensive procedures involving ion-exchange columns; and that it performs separation more rapidly and is more conducive to automation. 

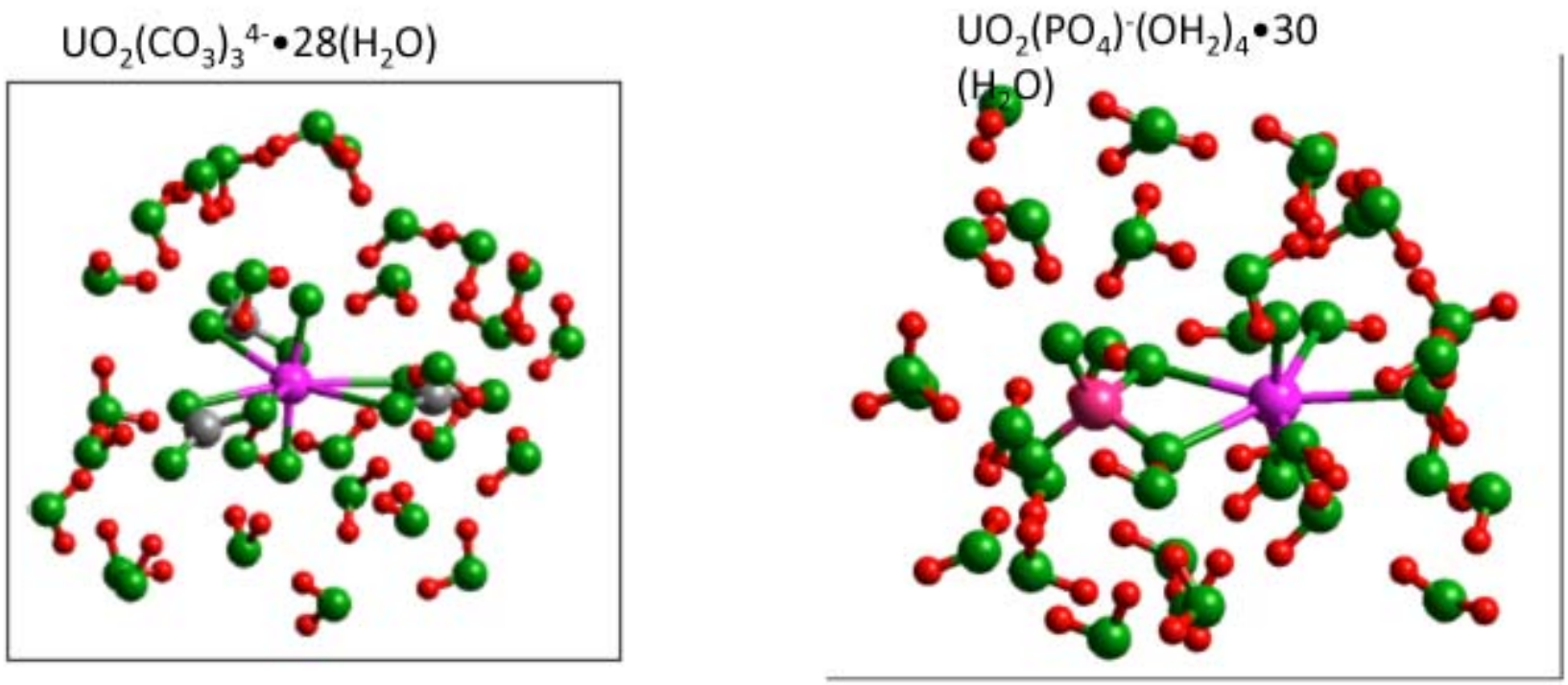

"Quantum mechanical calculation of aqueous uranium complexes: carbonate, phosphate, organic and biomolecular species" Kubicki, et al.

Quantum mechanical modeling of uranium ion complexes, such as those shown above, could be used as input to model electrophoretic mobilities. 